\title{
EL TRABAJO SOCIAL COMO PRÁCTICA PROFESIONAL Y SERVICIO SOCIAL COMO IMPULSOR DEL SABER
}

Fabiola Pedrero Jiménez*, Cecillia García Muñoz Aparicio*, Francisco Javier Jiménez Tecillo*, María del Carmen Navarrete Torres*.

Pedrero-Jiménez F., García-Muñoz Aparicio C., JiménezTecillo F.J., Navarrete-Torres M.C. El trabajo social como práctica profesional y servicio social como impulsor del saber. Hitos de Ciencias Económico Administrativas 2014;20 (57): 81-86.

\section{RESUMEN}

En este ensayo se demuestra la importancia del trabajo social (tanto en las prácticas profesionales como en el servicio social) como impulsor del saber y el hacer en las universidades, a través de la formación integral de los jóvenes universitarios, siendo su objetivo demostrar la importancia del mismo a través de una metodología de tipo documental descriptiva y cualitativa.

Palabras clave: Trabajo social. Práctica profesional. Servicio social.
Pedrero-Jiménez F., García-Muñoz Aparicio C., JiménezTecillo F.J., Navarrete-Torres M.C. The social work as professional practice and the social service as a promoter of knowledge. Hitos de Ciencias Económico Administrativas 2014;20 (57): 81-86.

\section{ABSTRACT}

In this paper it is shown the importance of social work (both professional practice and social service) as a promoter of knowledge and the doing in universities through the integral formation of college students, being its aim to demonstrate its importance through a documental descriptive and qualitative methodology.

Key words: Social work. Professional practice. Social service.

DIRECCIÓN PARA RECIBIR CORRESPONDENCIA: Correo electrónico: fabiola.pedrero@dacea.ujat.mx

$\mathbf{E}$

I trabajo social como parte de la práctica profesional y/o servicio social en las universidades, está considerado desligado de las mismas, aunque México es un país con una diversidad en sus regiones, un mosaico de una gran diversidad étnica, lingüística, socioeconómica y geográfica, un nivel variable de desarrollo caracterizado por una enorme heterogeneidad en lo geográfico, económico y cultural, marcado por fuertes variaciones en el grado de urbanización, en la disponibilidad de servicios y donde cohabitan diferentes estructuras domésticas, necesita que se formen estudiantes íntegros, con una moral fuerte basada en la práctica ética de las buenas costumbres. Es por eso que es de suma importancia examinar los programas de estudio en las universidades destacando su diversidad, analizando los roles y las funciones del trabajo social, la práctica profesional y el servicio social y su grado de importancia al desarrollarse en el ámbito académico, social y cultural.

*Profesores investigadores de la División Académica de Ciencias Económico Administrativas de la Universidad Juárez Autónoma de Tabasco.

Fecha de recibido: 20 de febrero de 2014. Fecha de aceptación: 24 de marzo de 2014. 
Los prestadores de este servicio puede realizar labores sociales o en la iniciativa privada para que puedan ser generadores de conocimientos, lo cual lesgenera la construcción de una identidad más sólida en el ámbito profesional (Ribeiro, López y Mancinas, 2007). En este punto, se puede destacar la importancia de que exista trabajo social dentro del servicio social y prácticas profesionales durante la aplicación de éstos lo cual conlleva a que exista una mayor conciencia social por parte de los estudiantes.

Ribeiro, López, y Mancinas (2007) comentan que la profesionalización del Trabajo Social en México, se describe como un proceso en donde posteriormente, se van analizando los roles y las funciones del trabajo social, afirmando el hecho de que esta profesión ha ocupado un lugar secundario en relación con otras profesiones de lo social, destacándose que una gran parte de los trabajadores sociales laboran en el sector de la salud; no obstante, cada vez más, ellos ocupan puestos en la empresa privada en los departamentos de recursos humanos. La última parte de este trabajo aborda algunas perspectivas de desarrollo del Trabajo Social y se incluyen algunas pistas sobre las necesidades en la enseñanza de esta disciplina, que podrían contribuir a la construcción de una identidad profesional más sólida, es por eso la necesidad de la integración el trabajo social con las prácticas profesionales y el servicio social ya que muchas veces en estos se hace una labor en la comunidad para beneficio de la misma y aunque el primero está constituido como una carrera, los otros son parte del desempeño de los estudiantes cuando llevan un avance de más del $50 \%$ de los créditos universitarios de algunas universidades.

Los escenarios para educar, reflexionar e investigar, han llevado a considerar a los espacios no convencionales que en el campo de la pedagogía social, son los que la educación se ha centrado en la atención de aquellos segmentos de la población considerados en desventaja social, especialmente de niños, niñas y adolescentes en situación de abandono, farmacodependientes, con problemas de conducta, así como de aquellos adultos en situación de privación y transición a la libertad. La educación en estos escenarios, implica traspasar las barreras del aula escolar formal para generar un nuevo prototipo que permita a los docentes tener una postura ética y actitud de apertura, de flexibilidad, con formación para conducir investigaciones, con conocimientos en metódica, como la concientización y en la toma de posiciones conscientes ante el mundo y su papel como ente social, es por eso la importancia que tienen los profesores en cuanto a el desarrollo de una conciencia social transformadora, siendo capaces de conocer, comprender y llevar a cabo acciones en particular (Peleteiro, 2009). En este punto, cabe destacar la importancia del servicio social y las prácticas profesionales en cuanto al trabajo social y su inclusión en éstos.

La práctica profesional y el servicio social constituyen la parte importante en algunas universidades donde se reviste la importancia, en el proceso formativo del estudiante, poniendo en juego los conocimientos adquiridos en la carrera bajo una continua interrelación entre ejercicio profesional y supervisión. Cuando se hace mención al concepto de práctica es inevitable plantear la relación teoría-práctica, no como se ha entendido tradicionalmente, como dos actos escindidos del conocimiento, sino como dos procesos complementarios mutuamente intervinientes, interactuantes y coexistentes. La constante tensión sugerida por la relación teoría-práctica se encuentra en permanente replanteamiento conceptual. La práctica 
académica tiene unos procedimientos técnicoinstrumentales definidos para su desarrollo desde el proceso formativo tanto en el sentido académico como en el administrativo en el contexto de la universidad, en tanto su objeto es social, en ésta también se vive un proceso cotidiano con el procedimiento técnicoinstrumental (Galeano, Rosero y Velazquez, 2011), mientras que el servicio social no es más que la devolución a la sociedad algo de los beneficios de haber estudiado, por lo que el trabajo social debe de ser considerado parte importante en éstos ya que está inmerso en los valores que marcan la estructura de un profesional.

El trabajo social, al igual que el servicio social y la práctica profesional al tomar como valor central el respeto a la persona en su dignidad y libertad, defiende también la aceptación y la autodeterminación como principios inherentes al ejercicio profesional, buscando en sus acciones la neutralidad, la actitud no judicial y evitar actuaciones definidas. Como consecuencia, la particularidad, singularización e individualización se consideran también principios básicos de la actuación profesional (García, 2007).

En lo que se refiere a las universidades, el contenido de los programas de licenciatura ha ido variando con el paso del tiempo, no sólo en función de las necesidades locales/regionales, sino también en relación con la ideología en juego que prevalece en cada institución académica; así como los organismos de acreditación haciendo que la formación y los perfiles de egreso de los estudiantes en México sean diferentes de en cada uno de sus lugares (Ribeiro, López y Mancinas, 2007). Es por ello, que la formación profesional es uno de los ejes de trabajo y discusión que se ha mantenido en Instituciones donde el trabajo social tanto de la práctica como del servicio social son importantes, lo cual demuestra la profunda preocupación y la importancia del tema en las Instituciones de Educación Pública ya que desde la experiencia docente en la formación universitaria de profesionales en estos, es aquel que tiene como propósito resaltar las características y cualidades en la formación profesional y tomar una pauta para la ejecución de acciones que concreticen un nuevo perfil de profesionales: técnicamente capaces, con altos conocimiento en su especialización, pero igualmente comprometidos con valores y principios éticos que aporten a la transformación del escenario actual donde prevalecen las desigualdades y las inequidades.

El trabajo social dentro de la práctica y el servicio social ha estado inmerso siempre en las referidas normas, principios, valores y criterios para poder diferenciar así lo prescrito, lo permitido, lo preferible y deseable de la acción profesional, ya que la ética está presente en muchos temas de conversación de los diferentes sectores sociales, se escribe y se debate sobre ella, se utiliza como arma arrojadiza de valoraciones de los adversarios, como estandarte en la defensa de programas y actuaciones individuales y grupales, se exhibe como medalla en los logros del «trabajo bien hecho", se pasea entre los juzgados, foros políticos y sociales (García, 2007).

Cabe destacar que tanto el servicio social como la práctica profesional deben de ampliar sus funciones y competencias en campos de trabajo aun reducidos, desconocidos, ya que al expandir conocimiento, hacen que a la hora de contratar al estudiante, este posea una mayor número de habilidades y competencias y esté totalmente calificado con funciones definidas (Fernández, 2008) teniendo como consecuencia que el 
estudiante posea una mayor conciencia de lo que sucede a su alrededor y no solamente en el área académica y laboral.

La participación social es un proceso político que contempla conocer los problemas que afectan a la sociedad, identificar las soluciones, plantear y desarrollar acciones en torno a la resolución de problemas y hasta evaluar la efectividad de las mismas. La participación social permitirá potenciar las capacidades políticas de las personas, grupos, comunidades y sociedad en general para acceder al poder político y participar activamente en la definición de un proyecto de sociedad socialmente inclusivo. La intervención profesional no se orientará exclusivamente a la conformación de estas organizaciones; en un proceso democrático y participativo, facilitará condiciones para fortalecer a las organizaciones sociales nuevas o las que ya tienen un cierto camino recorrido en esta materia (Chinchilla, 2006).

Una distinción teórica establecida por Bermudes, Celmira y Melgar (2006) parece interesante que el principio ocupacional y el administrativo como coordenadas del trabajo profesional, ya sea en lo estudiantes que se encuentran realizando la práctica o el servicio social, basados en la legitimidad de estatutos racionales, legales o científicos, no se llega a diferenciar totalmente. No obstante, se destaca la necesidad de que la sociedad acepte como legítimo el poder de una profesión y es importante que los cuerpos profesionales pueden obtener la jurisdicción en determinadas áreas de la división del trabajo, produciendo la autoconstrucción profesional y sus auto representaciones.
Fernandez (2008) afirma que en el campo educativo, en cuanto al trabajo social, se pueden proponer la actuación ante las necesidades sociales derivadas de la educación temprana, en la ayuda a la incorporación educativa, en el control y prevención de situación de riesgos; reforzando esta función educativa del trabajo social, se refuerza la dimensión formativa del la acción social. En el campo de la dependencia, podemos encontrar nuevos modelos de gestión de casos, de diseño de protocolos de gestión administrativa de prestaciones, de elaboración de guías y bases de datos; profundizando en la función gestora del trabajo social se ayudará a integrar en el proceso metodológico la denostada actuación administrativa de muchos profesionales. Mientras, en el campo de la mediación se asiste al desarrollo, bajo la dirección del postgrado universitario, de nuevos ámbitos de trabajo con familias, con comunidades locales y con sectores culturales en situación de conflicto o de potencial conflictividad social.

Actualmente, en algunas universidades existe el programa del servicio social comunitario, el cual brinda atención a las comunidades del Estado a través de proyectos multidisciplinarios, propuestos, planeados, organizados y ejecutados por los prestadores de servicio social, este se caracteriza por el trabajo multidisciplinario, lo que enriquece el aprendizaje de los estudiantes y posibilita una mayor atención a las necesidades de las comunidades y esto se relaciona con el trabajo social, en todos los aspectos.

\section{COCLUSIONES}

En la época actual existen muchas veces contradicciones entre los valores y la prácticas de la economía de mercado, la relación entre ciertos saberes profesionales y su reproducción del orden social, lo cual en algunas ocasiones trascienden en las prácticas profesionales y servicio social que llevan a cabo todos 
los de estudiantes que realizan estos fuera de la universidad.

El trabajo social es significativo en la implementación de la práctica profesional, y de la forma en que se interrelaciona entre lo académico y lo profesional, teniendo como base primordial el servicio social, ya que un estudiante primero realiza su servicio y posteriormente, la práctica debido a que es la forma en que se manifiestan los conocimientos aprendidos a lo largo del transcurso de su estancia en la universidad.

Los practicantes tanto del servicio social como de las prácticas profesionales, deben de poseer un conjunto de conocimientos orientados a un aprendizaje basado en la acción y la experiencia, las cuales se deben de conseguir a través del ejercicio de destrezas y competencias, interrelacionando tanto la teoría como la práctica. Se deben de evaluar los procesos en los que intervienen éstos y hacerles conciencia sobre la responsabilidad de cada una de sus actuaciones. De igual manera, es importante mencionar que el trabajo social muchas veces depende de las situaciones en las que se vean envueltos los estudiantes, ya que debido a que el servicio social comunitario ha dado excelentes resultados en la formación de estudiantes, donde adquieren una mayor conciencia sobre las condiciones de grupos vulnerables y su desarrollo en la sociedad y con esto se van desarrollando nuevas competencias, destrezas y habilidades en cuanto a la actuación de esto en relación a la ética profesional, debido a que se va haciendo conciencia sobre la situación económica actual del país.
REFERENCIAS

Bermúdez, M. M., Celmira, B. y Melgar, A. (2006). Tendencias actuales en el Trabajo Social uruguayo: hacia un campo profesional envejecido?. Revista Katálysis, 9(2). pp. 237-248

Chichilla, M.(2006) Acción colectiva e intervención profesional del Trabajo Social: límites y posibilidades para la construcción de ciudadanía. katálysis 9(2) pp.158-165.

Fernández, S. (2008). Identidad y formación en el trabajo social: desafíos corporativos del prácticum para la docencia y el ejercicio profesional. Portularia, VIII (2), pp. 153-164. Recuperado: www.redalyc.org/ articulo.oa?id=161015991008

Galeano; C.C., Rosero, K., Y., y Velásquez, P.A. (2011). Reflexiones y retos de la práctica académica en trabajo social. Recuperado: dintev.univalle.edu.co/revistasunivalle/index.php/ prospectiva/.../1401

García, B. (2007). Los profesionales del trabajo social y la ética profesional ante los nuevos retos $y$ necesidades sociales. Humanismo y Trabajo Social, 006 pp. 173-188

Heler, M. (coordinador), Casas, J.M., Cerletti A. y Digilio P. (2002). Filosofía Social y Trabajo Social. Elucidación de un campo profesional. Buenos Aires, Biblos.

Peleteiro, I.E. (2009). La práctica profesional no convencional en diversos espacios sociales a partir de la Pedagogía de la Autonomía de Paulo Freire. Retos y desafíos desde la Pedagogía Social. Revista de Investigación, 33(68).

Ribeiro, M., López, R.E. y Mancinas S.E. (2007). Trabajo social y política social en México. Revista Internacional de Ciencias Sociales y Humanidades, SOCIOTAM, XVII (2), pp. 175-200. 
Silva, Z. (2009). Escenario actual para la profesión de Trabajo Social, retos en la formación Académica. Ponencia presentada en el XIX Seminario Latinoamericano de Escuelas de Trabajo Social Universidad Católica. Santiago de Guayaquil. Guayaquil, Ecuador. 